\title{
Mechanism of flow-induced biomolecular and colloidal aggregate breakup
}

\author{
Breanndán Ó Conchúir and Alessio Zaccone* \\ Cavendish Laboratory, University of Cambridge, J. J. Thomson Avenue, Cambridge CB3 OHE, England, United Kingdom
}

(Received 25 January 2013; published 29 March 2013)

\begin{abstract}
The drift-diffusion equation is first solved analytically for the dissociation rate and lifetime of a biomolecular or colloidal dimer bonded by realistic intermolecular potentials, under shear flow. Then we show using rigidity percolation concepts that the lifetime of a generic cluster formed under shear is controlled by the typical lifetime of a single bond in its interior. The latter, however, is also affected by collective stress transmission from other bonds in the aggregate, which we account for by introducing a semiempirical, analytical stress transmission efficiency $0 \leqslant \Gamma \leqslant 1$ calibrated on several simulation data sets. We show that aggregate breakup is a thermally activated process in which the activation energy is controlled by the interplay between intermolecular forces and the shear drift. The collective contribution to the overall shear drift term is dominant for large enough fractal aggregates, while surface erosion prevails for small and compact aggregates. The crossover between the two regimes occurs when $\Gamma N \simeq 2$, where both the number of particles in the cluster $N$ and the stress transmission efficiency $\Gamma$ depend on the aggregate structure through the fractal dimension $d_{f}$. The analytical framework for the aggregate breakup rate is in quantitative agreement with experiments and can be used in future studies in the population balance modeling of colloidal and protein aggregation.
\end{abstract}

DOI: 10.1103/PhysRevE.87.032310

PACS number(s): 82.70.Dd, 62.20.-x, 61.43.Hv

\section{INTRODUCTION}

Large molecules exhibiting Brownian motion in a liquid solvent, like proteins, nanoparticles, and quantum dots, invariably interact via complex force fields due to dispersion (London-van der Waals) forces, hydrophobic interactions, and electrostatics [1]. The self-assembly of these colloidal particles is therefore a result of the microscopic interplay between the interactions and the diffusive transport. All these aggregation states, however, are studied in static environments which are macroscopically in equilibrium or close to equilibrium [2-5]. The phenomenology is completely different as soon as the environment is macroscopically perturbed and driven out of equilibrium such as under an externally imposed flow, which is ubiquitous in natural and technological processes [6-8]. The static picture becomes not just an idealization but simply inapplicable. For example, equilibrium aggregation pathways are forbidden under flow because we are always away from detailed balance and only kinetic theory can be used to rationalize the observations [9]. These difficulties are currently hampering our understanding and control of protein aggregation in cellular environments, where cytoplasmic flow is likely to affect the aggregation mechanism [10]. The same problems arise in biotechnology, where biomolecules are processed in flow apparatuses [11]. In the emerging field of microfluidics, due to the channel cross sections being of orders of magnitude close to the macromolecular and colloidal sizes, the effect of flow on the molecular and colloidal dynamics cannot be neglected in many instances [12]. Finally, in the context of nanomaterials, external flow offers the unprecedented opportunity of developing microscopically controlled mechanical manipulation of nanoparticles to tune and direct their self-assembly on large scales [13].

Here we present a theoretical analysis which constitutes a key step forward toward the realization of this program.

*az302@cam.ac.uk
Analytical approaches in the past have focused on the continuum description of the solid aggregates to calculate the flow-induced rupture either in terms of material yielding under the shear stresses [14] or, more recently, in terms of fracture driven by crack propagation [15]. The limitation intrinsic to continuum approaches is clear: by neglecting both interparticle interactions and Brownian motion, they cannot capture the microscopic interplay between diffusion, shear, and intermolecular interactions, which controls the aggregate's fate in shear, i.e., whether the aggregate can grow further or breaks apart under a given shear rate. Here we take an approach which is completely different. We start from first principles, namely, from the drift-diffusion equation governing the dynamics of the individual Brownian particles in the aggregate with realistic interactions, and then construct a framework which allows us to account for the collective flow-induced stress transmission into the aggregate, by using concepts from different fields such as rigidity percolation and stochastic rate theory. In this way, an analytical theory is developed which is able to quantitatively describe and rationalize experimental and numerical observations.

\section{DIMER BREAKUP RATE}

Let us start by considering the case of a dimer, that is, two particles or molecules bonded by intermolecular forces, in a flow field. We consider the most general case of intermolecular interactions between two spherical molecules or colloidal particles composed of both attractive and repulsive components. The case of pure attraction can be easily inferred by simply neglecting the electrostatic repulsion. In our calculations we consider an attractive van der Waals potential $V_{\text {VDW }}$, a repulsive screened-electrostatic (electric double-layer) potential $V_{e}$, and a steep hard-core repulsive potential $V_{\mathrm{HC}}$. The expressions for $V_{\mathrm{VDW}}$ and $V_{e}$ can be found in the textbooks [16], while for $V_{\mathrm{HC}}$ we use an expression from [17]. 
The dynamics of two Brownian particles in the presence of drift terms, that may comprise intermolecular interactions as well as an external field (such as the flow imposed on the suspension), is governed by the Smoluchowski diffusion equation [18] for the probability density $\rho$ of finding a center to center separation of $\vec{r}$ between both particles:

$$
\frac{\partial \rho}{\partial t}+\vec{\nabla} \cdot[-D \vec{\nabla} \rho+\vec{A} \rho]=0,
$$

where $D=k_{B} T / b$ is the particle diffusion coefficient which is related to the Stokes friction coefficient $b=6 \pi \mu a$ through the Stokes-Einstein relation ( $\mu$ is the solvent viscosity with $a$ being the particle radius). $\vec{A}$ is the overall drift term. By the definition of the stationary current $\vec{J}$, we recover the continuity equation $\partial \rho / \partial t+\vec{\nabla} \cdot \vec{J}=0$. At steady state, the continuity equation dictates that the stationary current of probability density over a spherical surface is $J=4 \pi r^{2}(-D \partial \rho / \partial r+$ $A \rho)$, where $A$ is the radial component of the drift field.

Now $\hat{r}$ is the unit vector from the center of one particle to the center of the other bound particle, i.e., along the radial coordinate $\vec{r}=(h+2 a) \hat{r}$. Clearly, only the current along this direction matters in the dissociation of the bond between two particles. The drift term in the presence of both intermolecular forces and an external flow reads as $A=-b(\partial V / \partial r)+b v_{r}$. Here $v_{r} \equiv \vec{v} \cdot \hat{r}$ is the radial component of the relative velocity that the two particles acquire with respect to each other due to the imposed flow. The radial current becomes in this case equal to the following expression:

$$
J=4 \pi r^{2}\left[-D(\partial \rho / \partial r)-b(\partial V / \partial r) \rho+b v_{r} \rho\right] .
$$

In polar coordinates we integrate over all angles to find this radial current across a spherical cross section. Only those regions of the solid angle where the flow drives the particles apart are considered in order to calculate the dissociation rate. As we are going to see below, noncentral tangential forces between particles are also present [19] and are essential for the mechanical stability of the aggregate under flow [20], although they do not directly contribute to the two-particle dissociation rate as they do not have components along $\vec{r}$.

Without loss of generality, we consider axisymmetrical extensional shear flow where the flow velocity $\mathbf{v}$ is given in Cartesian coordinates by $\mathbf{v}(x, y, z)=\dot{\gamma}[-x / 2,-y / 2, z]$, with $\dot{\gamma}$ signifying the shear rate, since this is a prototype of more complex flows such as cytoplasmic flows in the cells and the laminar (sub-Kolmogorov) flows inside turbulent eddies [21,22]. Results for simple shear $[\mathbf{v}(x, y, z)=\dot{\gamma}[y, 0,0]]$ differ only by a numerical prefactor of order unity, as we are going to show below. Under the assumption of weak coupling between the flow field and the density field, $\rho(r)$ and $v_{r}$ are relatively uncorrelated over the solid angle (one should recall that $v_{r}$ also depends on the polar angle of the vector $\hat{r}$ ). Hence, $\left\langle\rho v_{r}\right\rangle \approx\langle\rho\rangle\left\langle v_{r}\right\rangle$, where \langle\rangle indicates the angular average restricted to the regions of the solid angle where the relative velocity imparted by the flow acts to separate the particles. In general, we have

$$
\begin{aligned}
\left\langle v_{r}\right\rangle & =\frac{1}{4 \pi} \int_{\Omega} \dot{\gamma} r[1-\mathcal{A}(r)] f(\theta, \phi) \sin \theta d \theta d \phi \\
& =\lambda \dot{\gamma} r[1-\mathcal{A}(r)],
\end{aligned}
$$

with $\dot{\gamma}$ being the shear rate and $\mathcal{A}$ being the two-body hydrodynamic screening function which can be found in closed form for both extensional and simple shear flows [23]. $f(\theta, \phi)$ contains the dependence of the radial velocity on the polar and azimuthal angles and differs depending on the flow geometry. For instance, in the case of extensional flow we have $f(\theta, \phi)=$ $3 \cos ^{2} \theta-1$, which depends only on the polar angle. The integral is taken over the restricted set $\Omega$ of regions in the solid angle where the relative radial flow velocity (and the associated force) is positive, pushing the two particles away from each other. $\lambda \equiv \frac{1}{4 \pi} \int_{\Omega} f(\theta, \phi) \sin \theta d \theta d \phi$ is a geometric prefactor resulting from the restricted angular integral which thus depends uniquely on the flow geometry, i.e., $\lambda=1 /(3 \sqrt{3})$ for extensional shear flow and $\lambda=1 /(3 \pi)$ for simple shear flow.

With a purely algebraic manipulation, we can rewrite Eq. (2) as [24,25]

$$
J=-4 \pi r^{2} D e^{-V_{p} / k_{B} T} \frac{d}{d r}\left[e^{V_{p} / k_{B} T} \rho\right],
$$

where $V_{p} \equiv \int_{0}^{r} A d s$ is the primitive integral of the generalized drift $A$ introduced above. Following the Kramers procedure, we integrate Eq. (4) between $r^{*}$, a generic point near the potential minimum, and $C$. Here $C$ is some point far away on the radial axis. Since the probability density becomes negligible at $r=C$, because the effective potential has a large negative value there due to the drift term and the negative (volume-entropy) logarithmic term, we can express the constant current as

$$
J=\frac{e^{V_{p}\left(r^{*}\right) / k_{B} T} \rho\left(r^{*}\right)}{a^{-2} \int_{r^{*}}^{C} \frac{e^{V_{\text {eff }}(r) / k_{B} T}}{4 \pi D} d r},
$$

where the effective potential is given by

$$
V_{\mathrm{eff}} \equiv V-b \int_{0}^{r}\left\langle v_{r}\right\rangle d s-2 k_{B} T \ln (r / a) .
$$

This effective potential, as is going to be made clear below, maps our three-dimensional (3D) problem onto an effectively one-dimensional (1D) problem but leaves the physics unaltered. The logarithmic term is necessary to recover the metric factor $r^{-2}$ in the integral of Eq. (5), such that one can recover Eq. (2) upon going backward in the transformation. The integral in Eq. (5) is indefinite, because it is the primitive integral (or antiderivative), and the integration constant is chosen equal to zero such that we recover the case with no flow when $v_{r}=0$. $V_{\text {eff }}$ for realistic intermolecular interactions is plotted in Fig. 1. It is important to realize that the maximum (i.e., the effective energy barrier) is still present even when electrostatic repulsion is absent and actually even in the absence of shear. In the latter case the maximum is due to the entropy of dilution, because of the degrees of freedom gained by the particles upon breaking up the bond [25]. This contribution is qualitatively crucial for purely attractive interactions, but it is quantitatively small compared to the other contributions in our case.

The steady-state probability density inside the attractive well is given by the stationary Boltzmann distribution by means of the quasi-steady-state approximation in the well, $\rho(r)=\rho\left(r^{*}\right) e^{-\left[V_{p}(r)-V_{p}\left(r^{*}\right)\right] / k_{B} T}$.

Thus the probability of finding the particle in the 3D well is given by integrating the density over a spherical shell of this 


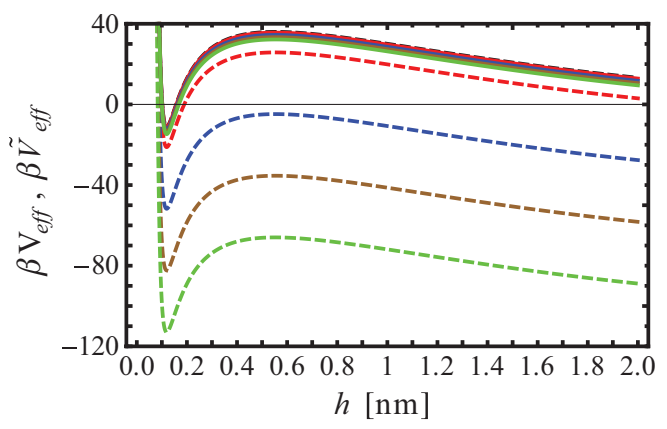

FIG. 1. (Color online) Plot of dimensionless $\beta V_{\text {eff }} \equiv V_{\text {eff }} / k_{B} T$ (solid) and $\beta \widetilde{V}_{\text {eff }} \equiv \widetilde{V}_{\text {eff }} / k_{B} T$ (dashed) as functions of the interparticle separation while varying the shear rate. From top to bottom, $\dot{\gamma}=$ $0 / 10^{3} / 4 \times 10^{3} / 7 \times 10^{3} / 10^{4}$. The calculations are shown for $a=$ $45 \mathrm{~nm}, d_{f}=2.9, R_{g}=450 \mathrm{~nm}, \sigma_{\mathrm{HC}}=2.08 \times 10^{-10} \mathrm{~m}$ (hard-core radius in the Born hard-core short-range repulsion [17]), $\psi_{0}=$ $0.05 \mathrm{~V}$ (surface potential), $T=298.15 \mathrm{~K}, \epsilon=7.09 \times 10^{-10} \mathrm{~F} / \mathrm{m}$ (water permittivity), $b=8.48 \times 10^{-10} \mathrm{~Pa} \mathrm{~m} \mathrm{~s}, n=67 \mathrm{~mol} \mathrm{~m}^{-3}$ (salt concentration), and $A=1.33 \times 10^{-20} \mathrm{~J}$ (Hamaker constant).

well:

$$
\rho_{e q}=\rho\left(r^{*}\right) e^{V_{p}\left(r^{*}\right) / k_{B} T} a^{2} \int_{A}^{B} e^{-V_{\text {eff }}(r) / k_{B} T} 4 \pi d r,
$$

where $A$ is a point to the left of the minimum and $B$ is a point to the right. Upon taking $C=\infty$, the mean firstpassage time is given by Kramers theory as $\tau=\rho_{e q} / J$. When both the minimum and the maximum are sharp, using the standard saddle-point method [26] to approximate the integrals analytically to quadratic order both near the well bottom and near the barrier top in the integrals appearing in $\rho_{e q}$ and $J$, respectively, we obtain the lifetime of the bond in shear as

$$
\tau=\frac{2 \pi b \exp \left[\beta V_{\mathrm{eff}}\left(r_{\mathrm{max}}\right)-\beta V_{\mathrm{eff}}\left(r_{\mathrm{min}}\right)\right]}{\sqrt{-V_{\mathrm{eff}}^{\prime \prime}\left(r_{\max }\right) V_{\mathrm{eff}}^{\prime \prime}\left(r_{\min }\right)}},
$$

where $r_{\min }$ and $r_{\max }$ represent the coordinates of the potential minimum and maximum and $\beta=1 /\left(k_{B} T\right)$. The flow-induced breakup rate is given by the inverse of the bond lifetime, $\kappa=$ $1 / \tau$.

\section{AGGREGATE BREAKUP}

\section{A. Rigidity-transition analysis of flow-induced colloidal aggregates}

After having laid down an analytical tool to calculate the breakup rate of a colloidal dimer under flow, let us now consider the breakup of an aggregate of many particles or molecules. In the most general case, the aggregate is defined by the number of constituent particles or molecules $N$ which is related to its radius of gyration $R_{g}$ via the mass-scaling relation $N=\left(R_{g} / a\right)^{d_{f}}$, where $d_{f}$ is the so-called fractal or mass-scaling exponent of the cluster [27]. In flow-induced aggregation, typical values of $d_{f}$ are in the range of 2.4 to 2.8 [7]. These values are significantly larger than the values found in diffusion-limited colloidal aggregation without flow $\left(d_{f} \simeq\right.$ $1.7-2)[16,28]$, the reason being the need for the aggregate to restructure (becoming denser) while growing in order to be able to withstand the flow stresses [29]. In the absence of a theory for $d_{f}$, this is the only phenomenological parameter in our analysis. It is worth recalling that in any case with $d_{f}<3$ the density of particles within the aggregate decays from the center of mass of the aggregate toward the outer shells as a power law of the size $\phi \sim\left(R_{g} / a\right)^{-d_{f}}$, where $\phi$ is the average packing fraction of molecules/particles [28]. This fact implies that the average packing fraction of larger aggregates is lower than the average packing fraction of smaller aggregates with the same $d_{f}$.

Computer-generated colloidal aggregates formed in flow conditions can be analyzed to obtain a relation between the average number of bonds per particle $z$, the radius of gyration $R_{g}$, and the structural parameter $d_{f}$. Analyzing Stokesian simulation data of flow-induced colloidal aggregates with both central-force and tangential interactions between colloids recently presented in the literature [30], we applied standard nonlinear least squares fitting procedures to obtain the following phenomenological relationship (with a confidence level of $95 \%$ and $R^{2}=0.988$ ) expressing $z$ as a function of $R_{g}$ and $d_{f}$ :

$z=0.532+0.776 d_{f}+0.000192 d_{f}^{6.462 d_{f}}\left(R_{g} / a\right)^{5.654-3.665 d_{f}}$.

In the definition of $z$ we took care in excluding dangling particles on the outermost shell of the cluster as they do not contribute to the internal rigidity. According to this relation, which is valid only in the regime of typical $2.4<d_{f}<2.8$, well-developed $\left(10^{2}<N<10^{4}\right)$ flow-induced aggregates, we have $2.4<z<2.8$, and $z$ increases with $d_{f}$. The decrease of $z$ upon increasing $R_{g}$ becomes less pronounced upon increasing $d_{f}$, reflecting the increasing structural homogeneity of the cluster as $d_{f} \rightarrow 3$.

Interestingly, the limit $z=2.4$ coincides with the rigidity transition of disordered assemblies where particles are bonded via both bond-bending (tangential) and central-force interactions [31]. For $z$ values below this threshold, any particle assembly has zero shear rigidity. The rigidity transition is marked by the fraction $f$ of floppy modes becoming nonzero at the transition point. We recall that a floppy mode is a global deformation mode of a particle assembly (i.e., a set of interparticle displacements from the original or equilibrium positions) which can be excited with a vanishing applied stress [32]. An aggregate which possesses a finite fraction of floppy modes is unstable with respect to a macroscopic volume-preserving deformation, such as shear, in the sense that it can be deformed by applying a vanishing stress. Equivalently, one can say that the shear modulus $G$ of an aggregate possessing floppy modes is zero and that a rigidity vanishing transition with $G=0$ sets in under conditions in which the aggregate develops a finite fraction of floppy modes.

The fraction of floppy modes in the presence of central and bond-bending interparticle forces, $f=N_{f} / 3 N$, is given by $f=1-\frac{1}{3}\left[\frac{1}{2} z+(2 z-3)\right]$ [32]. Hence, $f=0$ at $z_{c}=2.4$. Clearly, Eq. (9) suggests that the fractal aggregates developed in shear flow are marginally stable to shear stress with values of $z$ just above 2.4. Hence the scission of a small number of bonds in the cluster interior leads to the development of floppy modes and to the cluster becoming deformable even with a vanishing applied stress. Since the stress acting on the aggregate is nonzero under the applied flow, it is to be expected 
that the floppy aggregate undergoes large deformations. Such large deformations lead, in turn, to the aggregate breakup. Based on this argument and on the above analysis, we conclude that the lifetime of shear-generated aggregates with $z \simeq 2.4$ is in good approximation controlled by the lifetime of a bond in the aggregate interior.

Therefore, the aggregate may break apart stochastically when a bond or, at most, a few bonds break up due to the drift-diffusion mechanism, leading to floppy modes, global loss of rigidity, and large deformations. This picture, however, is complicated by the fact that, upon growing, the total stress impacting on the aggregate increases due to the presence of more particles exposed to the flow which all transmit stress into the aggregate. Hence, the breakup rate of the aggregate must depend on the aggregate size as well.

Our next exercise is then to calculate the breakup rate of a bond inside the aggregate by accounting for this effect and for the structure-mediated stress transmission in the aggregate.

\section{B. Collective stress transmission}

In a fractal aggregate every bond between two particles is exposed to the flow and experiences an averaged radial force $b\left\langle v_{r}\right\rangle$ which tends to stretch it. Since every bond is connected with other bonds, the flow-induced force acting on a bond can be transmitted to other bonds by means of force chains. Virtually every bond, then, receives flow-induced forces from every other bond in the aggregate by means of the force chain ensemble. Of course, due to dissipation at the interparticle contacts and other irreversible phenomena which occur in reality, only a fraction of these forces are effectively transmitted to a given particle in the aggregate. The total force transmitted to any particle in the aggregate core by force chains treated as harmonic springs (the bonds), considering that springs connected in series with the same spring constant undergo the same displacement, is given by $z(N / 2) b\left\langle v_{r}\right\rangle$. Since the particle has on average $z$ bonds, the force that ideally would act on an individual bond in the absence of dissipation and other effects is $(N / 2) b\left\langle v_{r}\right\rangle$.

The stress transmission crucially depends on the aggregate structure and its hydrodynamic permeability. For diffusionlimited fractal aggregates $\left(d_{f} \approx 1.7\right)$, the stress transmission is effectively $1 \mathrm{D}$ along the fractal strands of the aggregate [33]. If the aggregate is compact, on the other hand, the number of bonds per particle $z$ is significantly higher, and this is true even in the outer shells. Since realistically the force transmission efficiency through an interparticle bond is never equal to 1 because of dissipation due to friction and other irreversible processes at the interparticle contacts, it is then clear that in compact nonfractal aggregates the stress communicated by the flow is likely to be dissipated nearby on the outer shells without reaching the core. This fact has been argued by Edwards and Ball [34] long ago, and it has been confirmed by computer simulations of colloidal aggregates in shear [35]. As a result, the total force communicated to the aggregate structure is transmitted to the interior with a transmission efficiency $0 \leqslant \Gamma \leqslant 1$, which we expect to be a function of $d_{f}$. Furthermore, since it is not realistic to assume that the flow field has the same intensity in the core as on the surface (it is lower in the core due to hydrodynamic screening [36]), the total force transmitted will also be lower due to this fact. Hence, $\Gamma$ takes into account both dissipation and many-body hydrodynamic screening, in a phenomenological way.

To calculate the drift contribution due to collectively transmitted flow stress in the aggregate, we consider the overall force transmitted. This contributes an additional term to the drift $A$ in the drift-diffusion equation. The total drift acting along the radial coordinate between two bonded particles in the aggregate interior then becomes $A=-(\partial V / \partial r)+b\left\langle v_{r}\right\rangle+$ $\Gamma(N / 2) b\left\langle v_{r}\right\rangle$. The effective potential associated with the drift due to the collective stress reads as

$$
\begin{aligned}
\widetilde{V}_{\mathrm{eff}} & =V_{\mathrm{eff}}-\Gamma b(N / 2) \int_{0}^{r}\left\langle v_{r}\right\rangle d s \\
& =V-b(1+\Gamma N / 2) \int_{0}^{r}\left\langle v_{r}\right\rangle d s-2 k_{B} T \ln (r / a),
\end{aligned}
$$

where the tilde is used to disambiguate this potential, which contains collective many-body effects related to the aggregate structure, from the potential in Eq. (4) which refers to two particles not belonging to any aggregate.

\section{Aggregate lifetime and breakup rate}

The mean lifetime of the aggregate in closed form then follows as

$$
\tau_{\mathrm{agg}}=\frac{2 \pi b \exp \left[\beta \tilde{V}_{\mathrm{eff}}\left(r_{\mathrm{max}}\right)-\beta \tilde{V}_{\mathrm{eff}}\left(r_{\min }\right)\right]}{\sqrt{-\tilde{V}_{\mathrm{eff}}^{\prime \prime}\left(r_{\mathrm{max}}\right) \tilde{V}_{\mathrm{eff}}^{\prime \prime}\left(r_{\mathrm{min}}\right)}} .
$$

The breakup rate of the aggregate is given by $k_{\mathrm{agg}}=1 / \tau_{\mathrm{agg}}$. Using these expressions, by imposing the vanishing of the overall activation energy barrier in the exponential, one can calculate the critical size $R_{g}^{*}$, under a given $\dot{\gamma}$, beyond which breakup becomes a fast process due to the vanishing of the activation energy barrier for breakup. Hence, aggregates with $R_{g} \ll R_{g}^{*}$ may not break up on the time scale of observation due to the energy barrier, whereas aggregates with $R_{g} \gg R_{g}^{*}$ are unstable and break up instantaneously. In typical experiments or simulations of shear-induced colloidal aggregation, one measures the size distribution of aggregates. At steady state in the aggregation process, defined as the kinetic equilibrium where the average aggregate size becomes constant with time (because the aggregation and breakup rates become equal), no aggregates with $R_{g}>R_{g}^{*}$ can be found in the system as their lifetime is negligible. On the other hand, aggregation phenomena tend to generate larger aggregates with the consequence that the size distribution is controlled by the largest metastable aggregates and the measured average size is therefore close to $R_{g}^{*}$.

It follows that the above theory can be used to fit existing experimental and simulation data of the average steady-state size which can be approximately identified as $R_{g}^{*}$. The empirical stress-transmission efficiency $\Gamma$ can be used as a $d_{f}$-dependent fitting function to reproduce the dependence of the maximum stable size, $R_{g}^{*}$, versus $\dot{\gamma}$ found in different investigations for a broad range of $d_{f}$ [7,15,30,37]. From the fitting we obtained the following semiempirical stress-transmission function:

$$
\begin{aligned}
& \Gamma=\left(R_{g} / a\right)^{\alpha}, \\
& \alpha=-2.06491 d_{f}-0.0180344 /\left(3-d_{f}\right)+4.98585 .
\end{aligned}
$$


This expression has been found for flow-induced aggregates with $2.4 \lesssim d_{f} \leqslant 3$ and thus applies in this regime only. As one could expect, $\Gamma$ decreases upon increasing $d_{f}$ and ultimately vanishes at $d_{f}=3$. In terms of stress-transmission efficiency, this implies that the stress transmission to the aggregate interior becomes less efficient upon increasing $d_{f}$, i.e., upon increasing the aggregate density for a fixed size. This effect is due, in turn, to the increased dissipation in the outer shells of compact aggregates with respect to more fractal ones, where stress can be carried along quasi-one-dimensional fractal arms down to the core with much reduced dissipation. Also, many-body hydrodynamic screening limits the total force impacted onto the structure in denser assemblies. This formula, although phenomenological, fills a long-standing gap in the literature because no analytical approaches were hitherto available to assess the stress transmission in molecular and colloidal aggregates.

The effective potential $\tilde{V}_{\text {eff }}$ calculated in this way is plotted for typical conditions in Fig. 1 (dashed lines).

\section{DISCUSSION AND COMPARISON WITH EXPERIMENTAL DATA}

\section{A. Aggregate breakup rate}

Let us now discuss the predictions of Eq. (11) with $\Gamma$ defined by Eq. (12). In Fig. 2 we plot the breakup rate of aggregates as a function of the shear rate for realistic colloidal potentials. Two well-separated regimes are visible. For shear rates sufficiently low that the stress term $b(1+\Gamma N / 2) \int\left\langle v_{r}\right\rangle \mathrm{d} r$ is negligible with respect to the intermolecular bonding energy, the breakup rate is practically independent of $\dot{\gamma}$. When the stress term becomes comparable to $V$, the argument of the exponential attains a small value and the exponential can be linearized. In this intermediate regime, the aggregate breakup rate depends on the shear rate according to the following law:

$$
\kappa_{\mathrm{agg}} \sim \frac{\dot{\gamma}}{1+\beta\left\{\Delta V-b(1+\Gamma N / 2) \lambda \dot{\gamma} \int_{0}^{\Delta r} r[1-\mathcal{A}(r)] d r\right\}},
$$

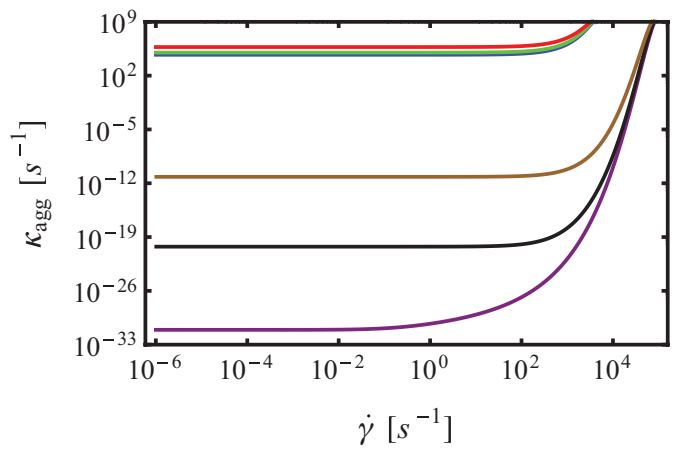

FIG. 2. (Color online) Plot of the breakup rate of an aggregate of radius of gyration $R_{g}=9 \mu \mathrm{m}$, colloid radius $a=45 \mathrm{~nm}$, and fractal dimension $d_{f}=2.7$. The bottom three curves correspond to initial potential barriers of 87,67 , and $47 k_{B} T$, where we employ a purely attractive interparticle potential in the first case. The top three curves highlight the same systems while neglecting two-body hydrodynamic interactions, i.e., setting $\mathcal{A}(r)=0$. where $\Delta V=V\left(r_{\max }\right)-V\left(r_{\min }\right)$ and $\Delta r=r_{\max }-r_{\min }>0$ refer to the intermolecular potential. Upon further increasing the flow intensity, the stress term in the exponential becomes comparable to the intermolecular interaction term and the exponential is no longer linearizable. Here the kinetics enters a new exponential regime where the breakup rate speeds up by several orders of magnitude upon further increasing $\dot{\gamma}$ :

$\kappa_{\text {agg }} \sim \exp \left\{-\beta \Delta V-\beta b(1+\Gamma N / 2) \lambda \dot{\gamma} \int_{0}^{\Delta r} r[1-\mathcal{A}(r)] d r\right\}$.

It is important to recall that many empirical models have been proposed in the past for the breakup rate of colloidal aggregates. Interestingly, some of these models assumed a power-law dependence for the breakup rate as a function of the shear rate, whereas other models, especially in the context of turbulent flows, assumed an exponential dependence [38]. The theory presented here resolves this long-standing conflict in the literature and shows that the power-law scaling applies to the regime where intermolecular forces dominate over the collective flow-induced drift, which tends to dissociate the bonds. It also shows that the exponential dependence on the shear rate applies only to the regime where the flow-induced drift is comparable to the intermolecular forces.

\section{B. Analysis of maximum stable aggregate size}

$R_{g}^{*}$ is estimated by putting $\tilde{V}_{\text {eff }}\left(r_{\text {max }}, R_{g}\right)-\tilde{V}_{\text {eff }}\left(r_{\text {min }}, R_{g}\right)=$ 0 and solving for $R_{g}$. This calculation has to be done numerically for the realistic colloidal potentials we are dealing with here. The result is a scaling relation between the stable $R_{g}^{*}$ value (beyond which the aggregate undergoes nonactivated, fast breakup) and the shear rate $\dot{\gamma}$. Hence, we need to solve $\beta \Delta V+\beta b(1+\Gamma N / 2) \lambda \dot{\gamma} \int_{0}^{\Delta r} r[1-\mathcal{A}(r)] d r=0$ for $R_{g}$ as a function of $\dot{\gamma}$.

Figure 3 compares the results of this calculation with recent well-controlled experimental data [30]. It is seen that the limiting size $R_{g}^{*}$ decays over a broad range of $\dot{\gamma}$ as a power law of the shear rate. In the comparison there are no free

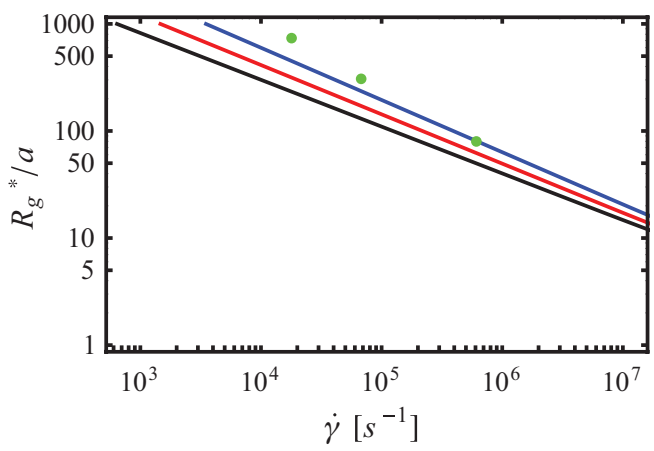

FIG. 3. (Color online) Log-log plot of $R_{g}^{*} / a$ as a function of $\dot{\gamma}$. From left to right, $d_{f}=2.5,2.6$, and 2.7. The circles are experimental data points [30] where $d_{f} \simeq 2.7$ was estimated by small angle light scattering. A purely attractive interparticle potential is employed using parameters matching the experimental conditions (polystyrene particles in water with screened electrostatic repulsion). The crossover to surface erosion occurs at a shear rate of order $10^{9} \mathrm{~s}^{-1}$, hence outside the range of the plot. 
parameters because we estimate the interparticle interaction using the parameters dictated by the experimental system (polystyrene colloidal particles in water with fully screened electrostatic interactions). Taking $d_{f}=2.7$, which is fixed by the experimentally measured fractal dimension [30], there is an excellent agreement between our predictions and the experimental data.

To obtain more insight into this scaling, let us consider the regime controlled by the collective stress transmission, $\Gamma N / 2 \gg 1$. Imposing the vanishing of the activation barrier we obtain

$$
R_{g}^{*} \sim \dot{\gamma}^{-\frac{1}{d_{f}+\alpha\left(d_{f}\right)}} \sim \dot{\gamma}^{p}
$$

where $\alpha\left(d_{f}\right)$ is given by Eq. (12). This result is important from several points of view. It provides a first-principles, microscopic theoretical explanation of the power-law scaling between aggregate size and shear rate that has been reported in many experimental and computational studies over the last decades. The physical meaning of this result is that the power law arises from the activation energy in Eq. (11), in which the flow-induced stress transmitted collectively by force chains into the aggregate, and mediated by the fractal aggregate structure, competes with the bonding energy. As the aggregate grows, the overall stress-induced drift that can be transmitted to an inner bond, without dissipation, increases with the aggregate size as $R_{g}^{d_{f}}$, but with intercontact dissipation this increase is slower and goes as $R_{g}^{d_{f}+\alpha\left(d_{f}\right)}$.

In the limit of nonfractal aggregates where the particle density is substantial throughout the aggregate, the stress transmission efficiency to the aggregate interior becomes very small because the stress is dissipated at the many interparticle contacts in the outer shells (which are as dense as the interior). Clearly, this is different from loose fractal aggregates, where the intercontact density is much lower in the outer shells, and the stress can be transmitted with lower dissipation due to the lower contact density. For this reason, in the limit of nonfractal aggregates, the collective drift term is small with respect to the local two-body shear drift term, $\Gamma N / 2 \ll 1$. As a consequence, bond breakup occurs locally, according to the two-body mechanism of Sec. II, directly on the surface where the shear stress is finite (in the $d_{f}=3$ limit the flow stress inside the aggregate is practically zero because the flow cannot penetrate into the dense compact aggregate). Hence, our theory predicts a crossover from a fragmentation mechanism, where the breakup is triggered by the dissociation of an inner bond under the action of stress transmitted through the structure, for $\Gamma N / 2 \gg 1$, into a surface erosion mechanism which is active for nonfractal aggregates in the regime $\Gamma N / 2 \ll 1$. This crossover is found as a function both of $d_{f}$ and of $R_{g}$, as the controlling factor $\Gamma N / 2$ depends on both $R_{g}$ and $d_{f}$.

In Fig. 4 we plotted the absolute value of the scaling law exponent $p$, which governs the scaling between size and shear rate, as a function of the fractal dimension [see Eq. (15)] in comparison with several sets of experimental and simulation data including Ref. [39,40]. Our model allows us to rationalize the dependence of the $R_{g}^{*}$ versus $\dot{\gamma}$ scaling law on $d_{f}$, according to the mechanism discussed above, in terms of the exponent $p$, over a broad range of $d_{f}$. From the plot it is evident that at sufficiently high $d_{f}$ there is a crossover from the breakup

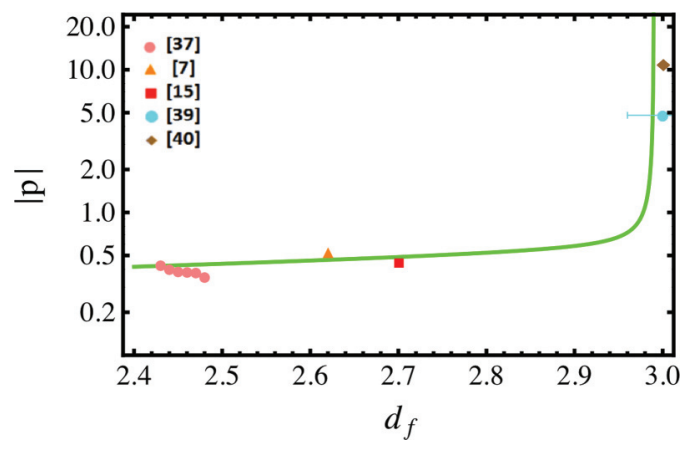

FIG. 4. (Color online) Plot of the absolute value of the power-law exponent $p$, as a function of $d_{f}$. The green (solid) curve indicates the prediction of this theory. Also included in this plot are measurements from different experimental and computational studies.

mechanism controlled by collective stress transmission, where the transmission efficiency is higher (reflected in small values of $|\alpha|$ and small values of $|p|$ ), into a regime controlled by surface erosion (large values of $|p|$ ), where stress transmission is very low due to localized intercontact dissipation, and breakup occurs on the surface due to the local effect of the shear. The crossover from the collective stress transmission to the erosive mechanism occurs when $\Gamma N / 2 \simeq 1$.

\section{Parameters used in the comparison}

If $d_{f}$ and the colloidal suspension parameters are available from experimental characterization, there are no freely adjustable parameters left to fit our analytical predictions against the experimental data in Fig. 3. As we mentioned in Sec. III, $d_{f}$ is a phenomenological parameter which is fixed by the experimental characterization of colloidal clusters in the experimental study. Thus, it is already fixed for each set of experimental data we wish to scrutinize our model against, provided that experimental $d_{f}$ values are available. $\Gamma$ is a semiempirical function whose form and coefficients, including $\alpha$, were calibrated on simulation data in the literature, and thus it cannot be altered for comparison with experiments. $\lambda$, as shown in Sec. II, is a geometric constant which is fixed by the flow type and is not adjustable. Therefore, there are no unconstrained model parameters with which comparisons with the experimental markers shown in Figs. 3 and 4 can be made. The values of the model parameters used in the comparison are univocally fixed by the experimental measurement $\left(d_{f}, a\right.$, interaction parameters), and by the flow type $(\lambda)$, while $\Gamma$ and $\alpha$ are universally fixed by the calibration procedure with simulation data and cannot be changed in the comparison with experiments.

\section{CONCLUSION}

In summary, we have presented a first-principles, microscopic analytical theory of macromolecular and colloidal aggregate breakup in shear flows. We first solved the Kramers escape rate problem for two arbitrarily bonded Brownian particles (dimer) in shear to calculate the dissociation rate and the mean lifetime of the dimer. Then we analyzed computer simulation data from the literature to show that aggregates formed in shear flow grow on the verge of marginal rigidity, 
which implies that they are close to the finite-floppy modes threshold. Hence, the flow-induced breakup of a very few bonds in the aggregate interior leads to the catastrophic collapse under shear of the whole aggregate along with the development of floppy modes. On this basis, we can therefore approximate the lifetime of an aggregate in shear flow by the lifetime of a bond in its interior. We account for the effect of many-body stress transmission and hydrodynamic screening from the aggregate structure through a semiempirical stresstransmission function which crucially depends on the aggregate size and fractal dimension. The final result is an analytical activation law expression for the breakup rate and lifetime. These are controlled by the competition, in the activation energy barrier for bond breakup, of the intermolecular bonding energy and the drift induced by the collectively transmitted hydrodynamic stress which works to dissociate the bond. With small aggregates, as well as in the limit of nonfractal, compact aggregates, our theory predicts a crossover from this collapse mechanism into surface erosion. In the latter regime, the flow cannot penetrate into the aggregate, but it can induce the removal of one particle at the time from the surface. Also, the analytical breakup rate expression can be used in future studies in the population balance modeling of colloidal and protein aggregation processes.

This theoretical framework allows us to rationalize many experimental observations in the literature. The mechanistic insights and analytical expressions provided here can serve as the basis to develop novel techniques for the controlled micro- and nanomechanical manipulation of nanoparticles in solution based on the calibrated use of shear flow. Also, it provides a rigorous basis for understanding the protein aggregation phenomena in physiological environments such as the cell, where cytoplasmic flows [10] can affect the growth of protein aggregates through the flow-induced breakup mechanism elucidated here.

\section{ACKNOWLEDGMENTS}

We wish to acknowledge Dr. Y. Harshe and Prof. M. Lattuada for making data of their published simulations available and for discussion. This study was supported by the Winton Programme (B.O.C.) and the Ernst Oppenheimer Fellowship (A.Z.). Many useful discussions with Prof. M. Morbidelli are gratefully acknowledged.
[1] J.-P. Hansen and I. R. McDonald, Theory of Simple Liquids, 3rd ed. (Academic, New York, 2006).

[2] P. J. Lu, E. Zaccarelli, F. Ciulla, A. B. Schofield, F. Sciortino, and D. A. Weitz, Nature (London) 453, 499 (2008).

[3] E. Zaccarelli, J. Phys.: Condens. Matter 19, 323101 (2007).

[4] A. Stradner, H. Sedgwick, F. Cardinaux, W. C. K. Poon, S. U. Egelhaaf, and P. Schurtenberger, Nature (London) 432, 492 (2004).

[5] P. J. Lu, J. C. Conrad, H. M. Wyss, A. B. Schofield, and D. A. Weitz, Phys. Rev. Lett. 96, 028306 (2006).

[6] R. G. Larson, The Structure and Rheology of Complex Fluids (Oxford University Press, New York, 1999).

[7] M. Soos, A. S. Moussa, L. Ehrl, J. Sefcik, J. Wu, and M. Morbidelli, J. Colloid Interface Sci. 319, 577 (2008); A. Zaccone, H. Wu, M. Lattuada, and M. Morbidelli, J. Phys. Chem. B 112, 6793 (2008).

[8] J. Mewis and N. J. Wagner, Colloidal Suspension Rheology (Cambridge University Press, Cambridge, 2012).

[9] A. Zaccone, H. Wu, D. Gentili, and M. Morbidelli, Phys. Rev. E 80, 051404 (2009).

[10] P. KhucTrong, J. Guck, and R. E. Goldstein, Phys. Rev. Lett. 109, 028104 (2012).

[11] V. Sluzky, J. A. Tamada, A. M. Klibanov, and R. Langer, Proc. Natl. Acad. Sci. USA 88, 9377 (1991).

[12] V. Fodera, S. Pagliara, O. Otto, U. F. Keyser, and A. M. Donald, J. Phys. Chem. Lett. 3, 2803 (2012).

[13] C. W. Wang, D. Sinton, and M. G. Moffitt, J. Am. Chem. Soc. 133, 18853 (2011).

[14] R. Sonntag and W. B. Russel, J. Colloid Interface Sci. 113, 399 (1986).

[15] A. Zaccone, M. Soos, M. Lattuada, H. Wu, M. U. Babler, and M. Morbidelli, Phys. Rev. E 79, 061401 (2009).
[16] R. Hunter, Foundations of Colloid Science Volume One (Clarendon Press, Oxford, 1987).

[17] D. L. Feke, N. D. Prabhu, and J. Adin Mann, Jr., J. Phys. Chem. 23, 5735 (1984).

[18] J. Dhont, An Introduction to the Dynamics of Colloids (Elsevier, Amsterdam, 1996).

[19] J. P. Pantina and E. M. Furst, Phys. Rev. Lett. 94, 138301 (2005).

[20] V. Becker and H. Briesen, Phys. Rev. E 78, 061404 (2008).

[21] G. K. Batchelor and J. T. Green, J. Fluid Mech. 56, 375 (1972)

[22] S. Melis, M. Verduyn, G. Storti, and M. Morbidelli, AIChE J. 45, 1383 (1999).

[23] M. Babler, J. Sefcik, M. Morbidelli, and J. Baldyga, J. Phys. Fluids 18, 013302 (2006).

[24] P. Haenggi, P. Talkner, and M. Borkovec, Rev. Mod. Phys. 62, 251 (1990).

[25] A. Zaccone and E. M. Terentjev, Phys. Rev. Lett. 108, 038302 (2012).

[26] H. A. Kramers, Physica 7, 284 (1940).

[27] P. M. Chaikin and T. C. Lubensky, Principles of Condensed Matter Physics (Cambridge University Press, Cambridge, 1995).

[28] W. B. Russel, D. Saville, and W. R. Schowalter, Colloidal Dispersions (Cambridge University Press, Cambridge, 2001).

[29] M. L. Eggersdorfer, D. Kadau, H. J. Herrmann, and S. E. Pratsinis, J. Colloid Interface Sci. 342, 261 (2010).

[30] Y. M. Harshe, M. Lattuada, and M. Soos, Langmuir 27, 5739 (2011)

[31] H. He and M. F. Thorpe, Phys. Rev. Lett. 54, 2107 (1985); A. Zaccone, Mod. Phys. Lett. B 27, 1330002 (2013).

[32] J. C. Phillips and M. F. Thorpe, Solid State Commun. 53, 699 (1985); A. Zaccone and E. Scossa-Romano, Phys. Rev. B 83, 184205 (2011). 
[33] Y. Kantor and I. Webman, Phys. Rev. Lett. 52, 1891 (1984).

[34] S. F. Edwards and R. C. Ball, Physica D 38, 88 (1989).

[35] M. Vanni and A. Gastaldi, Langmuir 27, 12822 (2011).

[36] M. Vanni, Chem. Eng. Sci. 55, 685 (2000).

[37] K. Higashitani, K. Iimura, and H. Sanda, Chem. Eng. Sci. 56, 2927 (2001).
[38] D. L. Marchisio, R. D. Vigil, and R. O. Fox, Chem. Eng. Sci. 58, 3337 (2003).

[39] S. P. Rwei, I. Manas-Zloczower, and D. L. Feke, Polym. Eng. Sci. 30, 701 (1990).

[40] N. D. Vassileva, D. van den Ende, F. Mugele, and J. Mellema, Langmuir 22, 4959 (2006). 